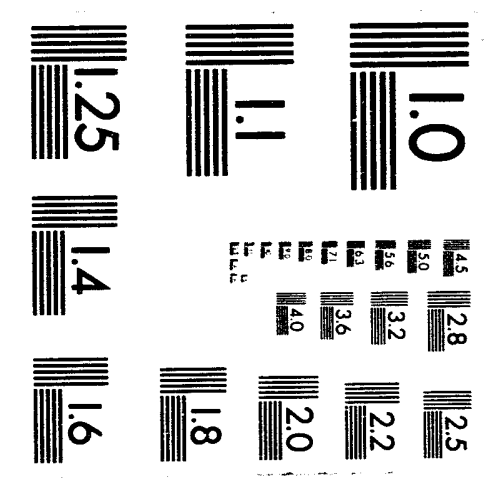



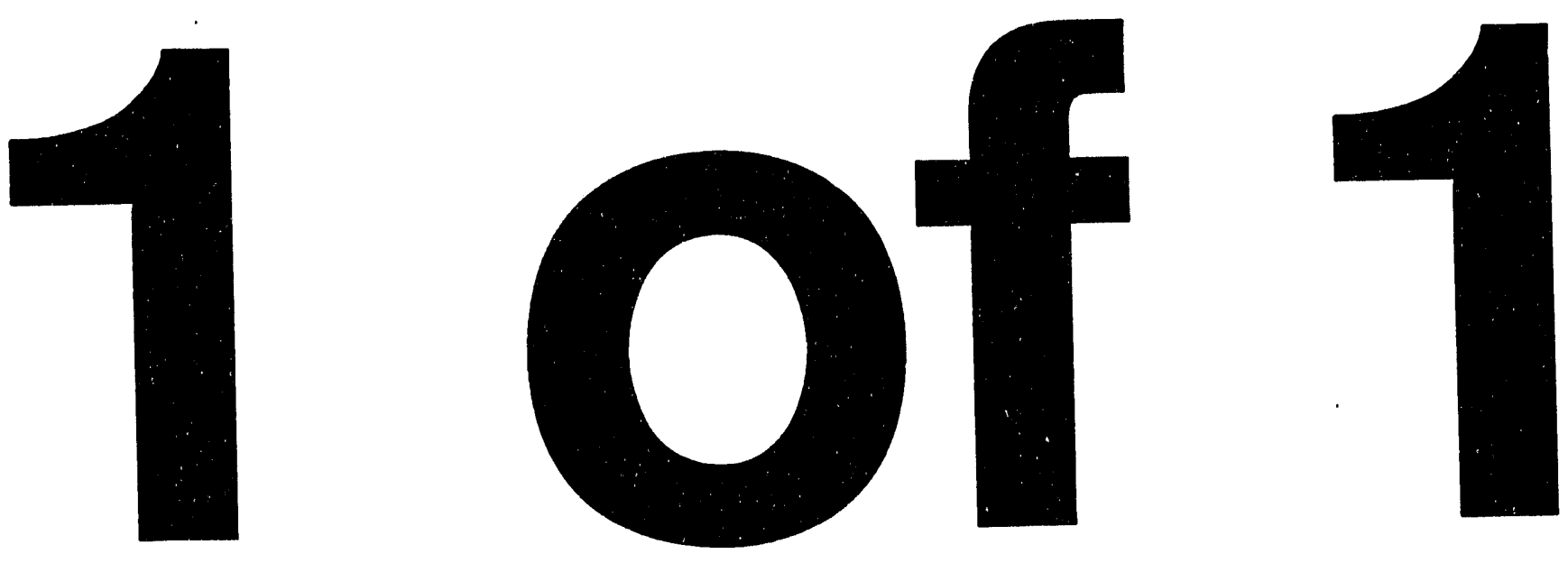


\title{
AN EVALUATION OF THE B\&W OWNERS GROUP BAW-10182 TOPICAL REPORT - JUSTIFICATION FOR INCREASING THE ENGINEERED SAFETY FEATURES ACTUATION SYSTEM ON-LINE TEST INTERVALS
}

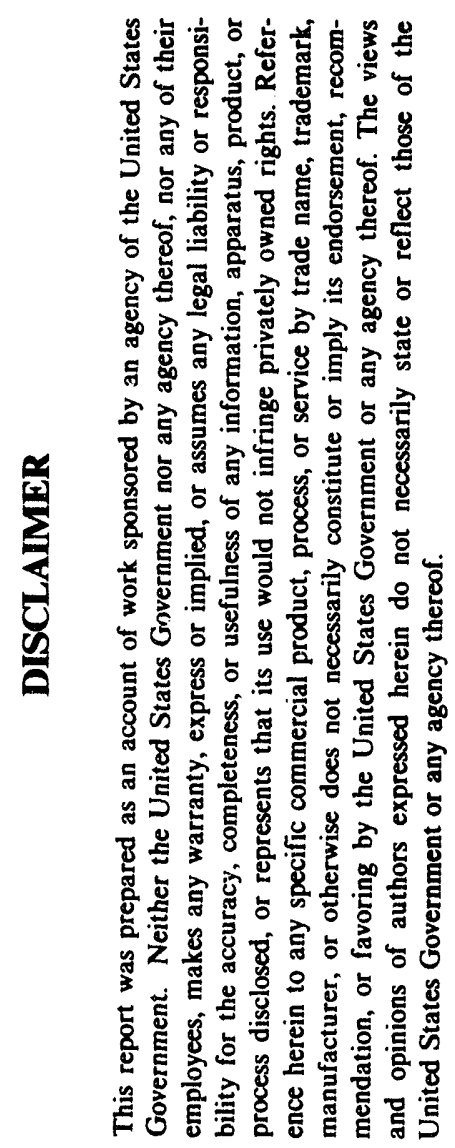

\author{
Curtis L. Smith \\ Jeff L. Hansen \\ September 1993 \\ Idaho National Engineering Laboratory \\ EG\&G Idaho, Inc. \\ Idaho Falls, Idaho 83415 \\ Prepared for the \\ Office of Nuclear Reactor Regulation \\ U.S. Nuclear Regulatory Commission \\ Washington, DC 20555 \\ Under DOE Idaho Operations Office \\ Contract No. DE-AC07-76ID01570 \\ FIN No. L1695, Task 13
}

TAC No. M-83011 


\begin{abstract}
This Technical Evaluation Report provides an evaluation of the Babcock and Wilcox Owners Group (B\&WOG) Technical Specifications Committee Topical Report BAW-10182, entitled, "Justification for Increasing Engineered Safety Features Actuation System (ESFAS) On-Line Test Intervals." This evaluation was performed by the Idaho National Engineering Laboratory in support of the Nuclear Regulatory Commission. The BAW-10182 report presents justification for the extension of on-line test intervals from the existing one-month interval to a threemonth interval for the ESFAS system. In the BAW-10182 report, the B\&WOG stated that "...the B\&WOG proposes to increase the ESFAS test interval from one to three months and concludes that the effect on plant risk is insignificant." The proposed extension was based upon risk-based [i.e., probabilistic risk assessment (PRA)] methods such as reliability block diagrams, uncertainty analyses, and timedependent system availability analyses. This use of PRA methods requires a detailed evaluation to determine whether the chosen methods and their application are valid in the context of the proposed test interval extension. The results of the evaluation agreed that the effect on plant risk is small if the ESFAS test interval is extended to three months for the ESFAS designs that were evaluated.
\end{abstract}

FIN No. L1695: Technical Assistance in Support of the Instrumentation and Control Systems Branch 


\section{CONTENTS}

ABSTRACT $\ldots \ldots \ldots \ldots \ldots \ldots \ldots \ldots \ldots \ldots \ldots \ldots \ldots \ldots \ldots \ldots \ldots \ldots \ldots \ldots$

ACRONYMS $\ldots \ldots \ldots \ldots \ldots \ldots \ldots \ldots \ldots \ldots \ldots \ldots \ldots \ldots \ldots \ldots$

1. INTRODUCTION AND REVIEW OBJECTIVES $\ldots \ldots \ldots \ldots \ldots \ldots \ldots \ldots$

2. BAW-10182 REPORT DESCRIPTION $\ldots \ldots \ldots \ldots \ldots \ldots \ldots \ldots \ldots \ldots \ldots$

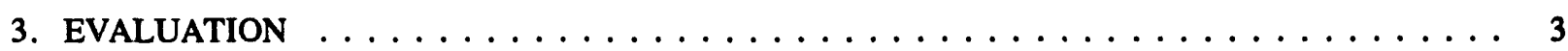

3.1 EVALUATION OF THE DESCRIPTION CHAPTER $\ldots \ldots \ldots \ldots \ldots \ldots \ldots$

3.2 EVALUATION OF MODELS USED FOR RELIABILITY EVALUATION $\ldots \ldots \ldots \ldots$

3.3 EVALUATION OF DATA FOR THE RELIABILITY EVALUATION $\ldots \ldots \ldots \ldots$

3.4 EVALUATION OF MODEL QUANTIFICATION $\ldots \ldots \ldots \ldots \ldots \ldots$

3.5 EVALUATION OF UNCERTAINTY ANALYSIS AND RESULTS $\ldots \ldots \ldots \ldots \ldots$

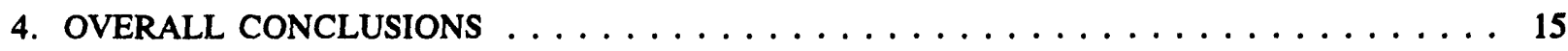

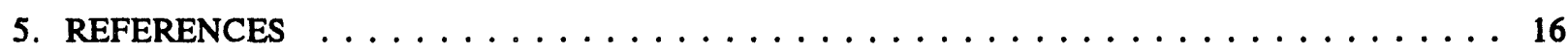




\section{ACRONYMS}

$\begin{array}{ll}\text { B\&WOG } & \text { Babcock and Wilcox Owners Group } \\ \text { CDF } & \text { cumulative distribution function } \\ \text { ESFAS } & \text { Engineered Safety Features Actuation System } \\ \text { MTTR } & \text { mean time to repair } \\ \text { NPRDS } & \text { Nuclear Plant Reliability Data System } \\ \text { NRC } & \text { Nuclear Regulatory Commission } \\ \text { PDF } & \text { probability density function }\end{array}$




\section{AN EVALUATION OF THE B\&W OWNERS GROUP BAW-10182 TOPICAL REPORT - JUSTIFICATION FOR INCREASING THE ENGINEERED SAFETY FEATURES ACTUATION SYSTEM ON-LINE TEST INTERVALS}

\subsection{INTRODUCTION AND REVIEW OBJECTIVES}

This report provides an evaluation of the Babcock and Wilcox Owners Group (B\&WOG) Technical Specifications Committee Topical Report BAW-10182, entitled, "Justification for Increasing Engineered Safety Features Actuation System (ESFAS) On-Line Test Intervals." 1 Included in the evaluation is a review of a supplemental letter containing additional information detailing specific issues in the BAW-10182 report. $^{2}$

B\&WOG report BAW-10182 proposes justification for the extension of on-line test intervals from the existing one-month interval to three-months for the ESFAS system. In the BAW-10182 report, the B\&WOG stated "...the B\&WOG proposes to increase the ESFAS test interval from one to three months and concludes that the effect on plant risk is insignificant." The proposed extension was evaluated by the B\&WOG using risk-based [i.e., probabilistic risk assessment (PRA)] methods such as reliability block diagrams, uncertainty analyses, and time-dependent system availability analyses. This use of PRA methods requires a detailed evaluation as to whether the chosen methods and their application are valid for the proposed test interval extension.

In addition to the general review (e.g., background information, references) of the BAW-10182 report, several specific areas were reviewed concerning the use of PRA methods. These areas were:

- A comparison of the component failure rates used in the report with failure data from the Nuclear Plant Reliability Data System ${ }^{3}$ (NPRDS) and other generic data bases such as the WASH-1400 data base and an EG\&G Idaho, Inc., generated generic data base.

- A comparison of the time-dependent analysis used in the report with the results of the Idaho National Engineering Laboratory time-dependent analysis using the FRANTIC ${ }^{4}$ computer code.

- An evaluation of the uncertainty values that were used for the component failure parameters.

- A determination of the acceptability and completeness of the general PRA methodology, including the reliability block diagrams, the time-dependent analysis, the uncertainty propagation method, the common-mode failure modeling, and the interpretation of the analysis results.

This technical evaluation looked at the probabilistic modeling methods that were used in the BAW-10182 report. Section 2 provides a brief overview of the technical contents for the report. Section 3 presents the detailed evaluation. of the report, including relevant comments related to technical issues contained in the report. Section 4 presents the overall conclusions of the technical evaluation. Section 5 provides the references that were used during the evaluation. 


\subsection{BAW-10182 REPORT DESCRIPTION}

This section provides a brief overview of the technical contents of the BAW-10182 report. The BAW-10182 report evaluated three different Babcock \& Wilcox ESFASs. Those three ESFASs were designed by Bailey (ANO-1 and Oconee), Gilbert (Crystal River-3), and Bechtel (Davis-Besse). The ESFAS for these power stations monitor applicable plant parameters such as reactor coolant pressure and reactor building pressure and have redundant subsystems which actuate the high pressure injection, low pressure injection, reactor building isolation, reactor building cooling, or reactor building spray systems.

The unavailability of each of the three ESFAS designs was modeled for the BAW-10182 report using reliability block diagrams. The modeling included two different cases: (1) the original onemonth ESFAS test interval and (2) a proposed three-month ESFAS test interval. The block diagrams incorporated common-mode failures. Cutsets were then generated from the block diagrams in order to be used for time-dependent core melt risk calculations. Uncertainty analyses were performed for the core melt risk calculations. These analyses concluded that the risk impact of increasing the ESFAS test interval from one to three months is insignificant.

The BAW-10182 report included descriptions of the computer codes, the models, the sources of data, the quantification of the data, and the uncertainty analysis methods. These areas are covered in detail in Section 3 of this review. 


\subsection{EVALUATION}

This section presents the review and evaluation of the BAW-10182 report. This section is divided into subsections corresponding to the major chapters of the report. In each subsection, pertinent issues or questions are discussed which relate to the information from the corresponding chapter in the report.

\subsection{EVALUATION OF THE DESCRIPTION CHAPTER}

Chapter 2 of the BAW-10182 report presents a general description of the hardware and testing requirements of the ESFAS systems. The proposed testing changes are also presented in this chapter. In general, the B\&WOG proposed changing the one-month test interval to a three-month test interval for those components which are tested on-line during reactor operation. This change could affect both analog and digital subsystems of the ESFAS. Changes to components outside the ESFAS system are not proposed in the BAW-10182 report, but this possibility is left open as a potential future option. If test intervals for components outside the ESFAS system are modified, the cumulative effect of changing test intervals for both the ESFAS components and other non-ESFAS components would need investigation. But, for the evaluation of the BAW-10182 report, it is appropriate to only investigate the risk change due to modifying the ESFAS test intervals.

\subsection{EVALUATION OF MODELS USED FOR RELIABILITY EVALUATION}

Chapter 3 of the BAW-10182 report presents an overview of the models and methods that were used to evaluate the reliability of the ESFAS systems. It was noted that the analysis methodology and data treatment for the BAW-10182 report is similar to that presented in the B\&WOG Topical Report BAW-10167 for the reactor trip system test interval extension. ${ }^{5}$ The analysis performed in BAW-10167 was reviewed and approved by the Nuclear Regulatory Commission (NRC). ${ }^{6}$ The differences between BAW-10167 and BAW-10182 are noted in Chapter 3 of BAW-10182.

One of the issues that was discussed in Chapter 3 of BAW-10182 is that, in the previous analysis (BAW-10167), the error factors on the independent failure data for the components may have been too low. ${ }^{1}$ Consequently, the error factors on the failure data for the components in BAW10182 were all increased to 10 . Without appropriate data and statistical analysis, a value of 10 for the failure data error factor is appropriate and should be considered an upper bound for the error factor value. It should be noted that the Reactor Safety Study ${ }^{7}$ (i.e., WASH-1400) stated that the "...relatively large error factor associated with instrumentation assessments reflect the wide variation in configuration from application to application," where the term "large error factor" is taken to be a value of 10 (i.e., the largest error factor listed for instrumentation in WASH-1400). Therefore, using an error factor of 10 is acceptable.

Modeling of common-mode failures was briefly discussed in Chapter 3 of BAW-10182. It was stated in the chapter that for the case where a common-mode failure rate could not be determined 
from the failure history, a subjective and "conservative" $\beta$-factor was used for the analysis. Chapter 4 of the BAW-10182 reports these "conservative" $\beta$-factors as having values of 0.1 . While a value of 0.1 for a $\beta$-factor is consistent with the current literature such as NUREG/CR-5801, ${ }^{8}$ this value may not be conservative as stated in BAW-10182. Nonetheless, NUREG/CR-5801 ${ }^{8}$ suggests the use of a $0.1 \beta$-factor for those components that did not have data available to estimate the $\beta$-factor. Thus, a $\beta$-factor of 0.1 is acceptable.

Reliability block diagrams were used in BAW-10182 to provide system failure cutsets. Appendices $\mathrm{A}, \mathrm{B}$, and $\mathrm{C}$ contained the block diagrams for the three ESFAS designs. These reliability block diagrams were reviewed for both correctness and completeness in modeling the ESFAS designs. In general, the block diagrams adequately represent their respective ESFAS designs. An appropriate level of detail is present in the block diagrams. Additional discussions on the reliability block diagrams are included in Section 3.3 of this report.

When performing the time-dependent system unavailability analysis, the mean-time-to-repair (MTTR) parameter may become one of the more important parameters, potentially impacting the overall system unavailability. For the BAW-10182 report, the MTTR parameter was set equal to the Technical Specification allowed outage time rather than the actual, historically-measured MTTR. By using the allowed outage time, the MTTR is set to its maximum possible value (assuming that the plant would be placed into a shutdown mode after the allowed outage time expires). According to BAW-10182, the allowed outage times for the ESFAS designs are:

"If failure of multiple analog channels is discovered, Technical Specifications require that the reactor be shutdown within a specified length of time to a mode where ESFAS operability is not required. For the Bailey plants, hot shutdown is required within 12 hours, and if not repaired within 48 more hours, then cold shutdown is required within 24 hours. For the Gilbert plant, hot shutdown is required within 13 hours for RC pressure or cold shutdown is required within 37 hours for radiation detection channels or hot shutdown is required within 13 hours for the other parameters. During this time, the reactor can return to power if the affected components are repaired and returned to service."

It was pointed out in BAW-10182 that single analog channel failures must generally be tripped (i.e., placed in a "safe" condition) within one hour and then repaired or replaced at the first opportunity. Thus, while using the Technical Specification allowed outage time for the MTTR for a particular component may seem to represent the maximum component outage time due to repair, this is only the case for when multiple analog channels are found failed. A single failed channel could be tripped until the next refueling outage for some of the ESFAS designs (those with two-outof-four coincidence). Consequently, the actual ESFAS reliability may be slightly different than that calculated in BAW-10182 if single failed channels are routinely kept in a tripped mode for periods of time longer than the multiple channel failure allowed outage time. One potential way to explicitly model this situation would be to perform the analysis using two different MTTR values. The first MTTR would represent the average repair time when only a single channel fails while the second MTTR would represent the average repair time when multiple channels fail. The digital subsystems do not have this concern since both single and multiple failures are given a particular allowed outage time. 
In order to evaluate the potential risk impact given that a single channel has failed, the analysis would have to evaluate the potential for an increase in spurious trips (since one of the ESFAS analog channels would be in a tripped state). It appears the potential increase in spurious trips was not evaluated for this situation. But, this potential increase in spurious trips due to a single failed channel could be negated by the decrease in spurious trips due to the reduced number of tests. Consequently, the core damage frequency impact from spurious trips due to the test interval increase is deemed to be negligible.

Aging, drift, and wearout effects to the components and subsystems in the ESFAS designs were not included in the BAW-10182 modeling. Reasons stated as to why these issues were not included in the modeling are:

- Wearout is not a significant issue for ESFAS monthly test interval extension.

- Each licensee should confirm that the drift will be within acceptable limits over the new test interval.

While not specifically quantified, the issues of wearout and aging do not warrant a substantial investigation. Typically, electrical devices such as the ESFAS exhibit a somewhat constant failure rate over the life of the component (as compared to mechanical devices). Increasing the ESFAS test interval to three months could potentially decrease the wearout failure contribution since the system is tested less often. As such, ignoring the effects of aging and wearout should not affect the conclusions of the analysis.

The issue of drift is not addressed due to the assumption that if a licensee increases the ESFAS test interval they will confirm that instrument drift stays within an acceptable limit. Increasing the ESFAS test intervals could allow instrument drift to degrade the ESFAS system whereas before increasing the test interval the instrument drift may not have reached the point of degrading the system. Therefore, it is important to minimize the potential for instrument drift. Each licensee will need to evaluate whether instrument drift is acceptable over the three-month interval.

Human errors were not modeled explicitly in the BAW-10182 report. Instead, the modeling of human errors was assumed to be accounted for in the common-mode and random failure rates. The justification given for not explicitly modeling human errors was that the human error failure contribution could not be separated out of the data that was used to quantify the common-mode and random failures. Given the potential difficulties in both reporting and evaluating data, the grouping of the human- and nonhuman-caused failures should not result in inaccuracies for the ESFAS unavailability calculations.

One statement in Chapter 3 reads, "Between tests, there is increasing unavailability according to the exponential relationship of reliability versus time..." This statement is true only for the case where the component hazard rate (or instantaneous failure rate) is assumed to be a constant. For most risk and safety analyses, the assumption is made that the hazard rate is a constant. For the 
analysis performed in BAW-10182, the assumption of a constant hazard rate for the components in question is valid.

When discussing the risk significance of ESFAS reliability, BAW-10182 stated that, "a secondary reason [why plant specific PRAs were not used] was to avoid bringing the generic applicability of plant-specific PRAs into question." The reliability block diagrams that were used for the analysis were not "generic" models and, instead, were tailored one of three particular designs. Using the appropriate ESFAS model with its associated plant specific PRA should not cause question as to whether the appropriate modeling method was selected. Instead, the method that was used in BAW-10182 was to take a particular plant design ESFAS and incorporate generic challenge events (i.e., initiating events) and recovery probabilities. This combination of plant-specific ESFAS modeling with generic challenge events and recovery probabilities raises the potential for underestimating the potential risk due to increasing the ESFAS test intervals. As a hypothetical example, if a particular plant experiences challenge events more often than the industry average while having less of a recovery potential than the industry average, the potential risk importance of the ESFAS system could be underestimated. But, since the models and model parameters are intended to represent a "typical" ESFAS and the risk increase was calculated as negligible for this system, bringing additional plant specific PRA information into the analysis would not change the overall results and conclusions of the analysis. Instead, the results represent an average B\&W ESFAS.

Chapter 3 discusses the various computer codes that were used to perform the analysis in BAW10182. Since these codes were either a public domain type code (e.g., FTAP) or were previously reviewed, it is assumed that the computer codes were used correctly, providing justifiable results (given that appropriate models are used for the analysis).

\subsection{EVALUATION OF DATA FOR THE RELIABILITY EVALUATION}

Chapter 4 of the BAW-10182 report addresses sources, development, and use of data for the analysis. In general, the report made use of operating experience whenever possible in order to quantify failure rates for the ESFAS components.

The source of data for analog sensors and instrument strings (for both random and commonmode failures) was NUREG/CR-3289. ${ }^{9}$ While NUREG/CR-3289 is somewhat dated (it was released in 1983) and only evaluated data collected for six years, the use of the data reported in the NUREG/CR-3289 report is adequate for the type of analysis performed. Additional operational experience data (since 1983) could be evaluated and added to the data in NUREG/CR-3289. But since NUREG/CR-3289 evaluated over 1,100 different Licensee Event Reports, it is probable that additional data will not significantly change the failure data that was presented.

The BAW-10182 report correctly used the data that was presented in NUREG/CR-3289 for most cases. One minor inconsistency that was noted was for Table 4-1 in BAW-10182. For Table 4-1, the row labeled "RB PRESSURE (DIGITAL) SWITCH" contains data for a non-lethal shock $(\mu)$ for a system size of six. This row should actually be labeled as pertaining to a system size of 
four (since NUREG/CR-3289 only lists data for system sizes of three, four, and eight for pressure switches). If the system size to be evaluated for common-mode failure events is actually six instead of four, the failure parameter to be used could be interpolated from the size-four and size-eight data. This interpolation would result in a slightly lower failure rate than that taken from the size four data. Consequently, the application of the size-four data for a size-six system results in a slightly conservative analysis (assuming that the pressure switches are actually a size-six system). The rest of the data in Table 4-1 appears to be correct.

The use of NUREG/CR-3289 data for the common-mode failure modeling appears to be correct. In general, the method that is used in the BAW-10182 report for common-mode failures appears to be appropriate and consistent with current methods ${ }^{8}$ of modeling potential common-mode events. But, one lapse in the common-mode modeling could be the fact that common-mode failures between different type systems was not quantified. BAW-10182 justifies this by stating, "B\&W experience indicates that there is no evidence supporting a common mode failure between two independent sensed parameters. Also, in NUREG/CR-3289, there were no events that failed channels of unlike parameters; only the probabilities of occurrence for common mode failures between channels of like parameters were estimated." It is true that NUREG/CR-3289 did not estimate common-mode failure probabilities for channels of unlike parameters. But this does not necessarily mean that commonmode failures of unlike channels is impossible. NUREG/CR-3289 can be used as an example of a potential common-mode failure between unlike channels; page C-16 briefly described two events that occurred at the Quad Cities 1 power plant on 4/15/1980. Both the reactor low water level switch and a pressure switch failed due to the same cause: instrument setpoint drift. (These events signify the potential importance of monitoring and managing instrument drift.) While these types of unlikesystem common-mode failures are rare, the potential exists for them to occur. But, the omission of these events from the modeling in BAW-10182 is not expected to alter the results of the analysis since these events occur very infrequently. For example, the failure event review of the B\&W operating history in the BAW-10182 report did not yield any unlike-system common-mode failures. ${ }^{1}$

In Chapter 4 of BAW-10182, it was stated that "when the failure history of the component showed no evidence of multiple failures..., it was necessary to apply engineering judgement and assume a conservative value for the beta and gamma factor, (i.e., $\beta=.1, \gamma=.5$ )..." While NUREG/CR-5801, Procedure for Analysis of Common-Cause Failures in Probabilistic Safety Analysis ${ }^{8}$ recommends a beta factor of 0.1 , it does not imply that this value for beta is a conservative value. Just because a system of components shows no evidence of multiple failures does not directly imply that the common-mode failure contribution is negligible (i.e., close to zero). For a system that demonstrates high reliability, failures may not appear very often. Consequently, the common-mode failures may not be evident unless data is collected for a long time (relative to the average time-to-failure) for a large number of like systems. This scenario may be evident in the data collected for the common-mode analysis in BAW-10182 since only two common-mode events were stated as occurring (one for the power supply modules and one for logic relays) yet the beta factors for the two events were listed as 0.4 and 0.25 (in Table 4-2), respectively. Since the calculated beta factors were larger than 0.1 and the number of recorded common-mode failures was small, this implies that the total number of recorded failures for the components in question was small. If the total number of recorded ESFAS component failures is small, it is expected that the number of 
common-mode failures is even smaller and may not be evident at all. But, as stated above, this lack of common-mode failures in a system with high reliability does not necessarily imply that a beta factor of 0.1 is conservative. In conclusion, it is correct to use a beta factor of 0.1 when data does not exist to quantify the beta factor, but it is not necessarily correct to state that the use of 0.1 for the beta factor is conservative when data is not available.

The BAW-10182 analysis used a constant, hourly failure rate for the ESFAS components instead of a demand failure probability. The use of an hourly failure rate instead of a demand failure probability was stated as being the conservative approach since all the available data was lumped into one parameter. An underlying assumption for the justification that the approach is conservative is that the failure probability for a particular ESFAS component is actually given by two parts, the demand and hourly failure contributions. Consequently, the approach could potentially be an accurate estimate (instead of conservative) if the demand failure contribution for a particular ESFAS component is negligible. Recent work for the NRC, NUREG/CR-5823, ${ }^{10}$ mathematically attempted to separate the demand and hourly failure contributions for a set of emergency diesel generator and motor operated valve failure data. One of the conclusions of NUREG/CR-5823 was that the motoroperated valve data showed that failures occurred almost exclusively due to hourly (i.e., standby) failures and that the demand failure contribution from the collected data was zero. As such, it may be premature to state that using only a hourly failure rate results in a conservative analysis. But, using an hourly failure rate instead of the combination of hourly and demand failure rates for the analysis would be either conservative or a best estimate. Consequently, the assumption of the hourly failure rate is adequate for the analysis.

The ESFAS challenging event frequencies (i.e., initiating events) that were listed in Table 4-3 of the BAW-10182 report were compared to initiating event frequencies from three of the NUREG/CR-4550 PRAs. ${ }^{11-13}$ The frequencies used in the analysis appear to be representative of typical frequencies used in other PRAs.

The random failure rates for the ESFAS components were compared to a generic failure rate database compiled by EG\&G Idaho ${ }^{14}$ and failure rate data from the NPRDS data collection system. Table 1 shows the comparison of the failure rates with the EG\&G database. As can be seen from the table, both the failure rates and error factors on the failure rates show close agreement. Some of the BAW-10182 failure rates are either a little higher or a little lower than the failure rates in the generic database, but they are reasonable given the uncertainty or the plant-specific nature (i.e., specific to B\&WOG plants for the digital components).

One item of note concerns the use of the failure rates in the analysis. In some risk analysis codes, an approximation is used to evaluate the probability of failure for a particular component. For a component with a constant failure rate $\lambda$ and a mission time of $t$, the exact expression for the failure probability in the mission time is: $P($ failure $)=1-\mathrm{e}^{-\lambda t}$. When the product of $\lambda t$ is less than 0.1 , then the exact expression for the failure probability can be approximated as $\mathrm{P}$ (failure) $\approx \lambda \mathrm{t}$. Reference 2 stated that the computer codes that were used for the analysis used the exact expression for the failure probability. The use of the exact expression rather than the approximation is appropriate for the analysis. 
Table 1. A comparison of the BAW-10182 random failure rates with a generic failure rate database.

\begin{tabular}{|l|c|c|c|c|}
\hline \multicolumn{1}{|c|}{ Component } & $\begin{array}{c}\text { BAW-10182 } \\
\text { Failure Rate } \\
\left(\mathrm{hr}^{-1}\right)\end{array}$ & $\begin{array}{c}\text { Error } \\
\text { Factor }\end{array}$ & $\begin{array}{c}\text { EG\&G generic } \\
\text { Database } \\
\text { Failure Rate } \\
\text { (hr-1) }\end{array}$ & $\begin{array}{c}\text { Error } \\
\text { Factor }\end{array}$ \\
\hline RB Pressure Sensor & $1.90 \mathrm{E}-06$ & 10 & $1.0 \mathrm{E}-06$ & 10 \\
\hline RC Pressure Sensor & $1.90 \mathrm{E}-06$ & 10 & $1.0 \mathrm{E}-06$ & 10 \\
\hline RB Pressure (digital) Switch & $7.70 \mathrm{E}-07$ & 10 & $1.0 \mathrm{E}-06$ & 10 \\
\hline Radiation Sensor & $4.50 \mathrm{E}-06$ & 10 & $5.0 \mathrm{E}-06$ & 10 \\
\hline BWST Level Sensor & $1.90 \mathrm{E}-06$ & 10 & $1.0 \mathrm{E}-06$ & 10 \\
\hline Instrument Strings & $3.10 \mathrm{E}-06$ & 10 & not available & - \\
\hline Trip Module & $1.60 \mathrm{E}-06$ & 10 & not available & - \\
\hline Output Module & $4.80 \mathrm{E}-07$ & 10 & $3.0 \mathrm{E}-06$ & 10 \\
\hline Logic Buffer Module & $5.08 \mathrm{E}-07$ & 10 & $3.0 \mathrm{E}-06$ & 10 \\
\hline Unit Control Module & $1.57 \mathrm{E}-07$ & 10 & not available & - \\
\hline Sequence Module & $3.49 \mathrm{E}-06$ & 10 & not available & - \\
\hline Power Supply & $5.35 \mathrm{E}-07$ & 10 & $1.0 \mathrm{E}-05$ & 10 \\
\hline Inverter & $2.58 \mathrm{E}-05$ & 10 & $5.0 \mathrm{E}-06$ & 5 \\
\hline Station Battery & $4.63 \mathrm{E}-06$ & 10 & $1.0 \mathrm{E}-05$ & 10 \\
\hline Relay (CR-3) (coil) & $1.01 \mathrm{E}-07$ & 10 & $5.0 \mathrm{E}-07$ & 10 \\
\hline Undervoltage Relay & $1.45 \mathrm{E}-08$ & 10 & $3.0 \mathrm{E}-07$ & 10 \\
\hline & $3.41 \mathrm{E}-07$ & 10 & $5.0 \mathrm{E}-07$ & 10 \\
\hline
\end{tabular}




\subsection{EVALUATION OF MODEL QUANTIFICATION}

Chapter 5 of the BAW-10182 report addresses both the time-dependent and time-averaged quantification of the analysis models. This quantification was performed through the use of computer codes developed (or modified) by B\&W. Since these computer codes are proprietary and were not available for use during this technical evaluation, an attempt was made to reproduce the quantification results using computer codes available to the evaluator (e.g., FRANTIC, ${ }^{4}$ IRRAS ${ }^{16}$ ).

The time-dependent quantification was performed for each of the three ESFAS designs for (1) each of the six challenging events and (2) an aggregate case including all challenging events. This quantification was performed for both the current one-month test interval and the proposed threemonth test interval. These time-dependent quantification runs are shown in Figures 5-1 through 5-5 in the BAW-10182 report. The figures appear similar to those that result from other time-dependent computer codes such as FRANTIC.

It was noted that the time-dependent analysis assumed that "...tested equipment is assumed to be returned to service failure free..." Previous analyses have shown that if the probability of imperfect testing or test-caused failures is on the order of 1-2\% then the overall system unavailability could increase by a factor of two or three. ${ }^{15}$ But, since the ESFAS has specified acceptance criteria in order to pass the test, and restoration is assumed to be independently verified, it is deemed that the probability test-caused failures or imperfect testing is negligible. If the probability of test-caused failures or imperfect testing is assumed to be negligible, then it would be expected that their contribution to the overall system unavailability would be negligible.

The time-averaged results were obtained by integrating over the time-dependent risk curve, which is the correct method of obtaining the "average" core-melt frequency over the time period of interest. The overall change in risk (called the delta risk) due to increasing the test interval from one to three months is calculated by subtracting the time-averaged result for the one-month test interval from the time-averaged result for three-month test interval.

On page 5-5 of the BAW-10182 report, it is stated that, "Some previous PRA-based studies were examined to determine their consensus on the contribution of ESFAS to risk, and to see if the risk-significance compared favorably with the ESFAS risk significance calculated in this study." References for the studies that were used for the comparison should be provided.

The results of the analysis provide answers in terms of the change in the core melt frequency. While the analysis of the core melt frequency investigates the changes in the ESFAS unavailability, additional information could have been provided if the change in system unavailability was also presented separately from the core melt frequency results. To investigate the potential change in ESFAS unavailability, the analysis for one of the ESFAS systems (the Bailey design) was duplicated as closely as possible. This duplication consisted of: (1) converting the reliability block diagrams into fault trees, (2) using the fault trees to generate system failure cut sets, (3) loading the cut sets into the FRANTIC time-dependent unavailability computer code, (4) evaluating the ESFAS unavailability using the parameters given by BAW-10182, and (5) performing sensitivity analyses 
on the test interval parameter. Figure 1 shows the results of the analysis duplication (i.e., those performed with the FRANTIC computer code). As can be seen from the FRANTIC analysis in Figure 1, the ESFAS unavailability increases by a factor of three when the test interval is increased to three months from one month.

Although the ESFAS unavailability increased by a factor of three (going from one to a threemonth test interval) when the BAW-10182 analysis was duplicated using FRANTIC, the change in the core melt frequency will not necessarily be a factor of three. For example, a particular component could have its overall outage time reduced $87 \%$, which may only decrease its associated system unavailability $45 \%$, which may only decrease the core melt frequency $4 \%$. The magnitude for these "trickle-down" effects are component-, train-, system-, or plant-specific. Consequently, for a particular system that does not contribute significantly to the overall core melt frequency, significant reductions in its unavailability may have negligible effects on reducing the core melt frequency. (Note: This implies that an increase in system unavailability may also have a negligible effect on increasing the core melt frequency, and is one of the motivations to develop and implement risk-based changes to Technical Specifications and testing intervals). To test the potential change in the core melt frequency, the ESFAS system failure probability was increased by a factor of three in the IRRAS $4.0^{16}$ electronic Oconee PRA database. (IRRAS is a PRA fault tree/event tree analysis computer code.) The change in the core melt frequency after increasing the ESFAS system failure probability was negligible.

The last paragraph in Chapter 5 refers to a Brookhaven National Laboratory report, NUREG/CR-5200, ${ }^{17}$ (which is actually reference 21 and not 20 in the BAW-10182 report) in order to support the analysis results that were obtained. While NUREG/CR-5200 did state that surveillance tests related to the ESFAS could be extended without affecting risk, it also stated (page 28) that, "...manufacturer-recommended test intervals should not be violated, if they are necessary to maintain the integrity of the component." While the manufacturer-recommended test intervals for most components are determined by deterministic means rather than probabilistic means, the manufacturer may have sufficient justification for recommending a particular testing interval. As such, attention should be given to test interval recommendations from the manufacturer of a particular system or component when attempting to modify test intervals.

Three comments concerning Table 5-1 of the BAW-10182 report are: (1) the number of significant figures for the core melt risk results are not justified, (2) round-off errors exist in the second decimal place when the one-month test interval results are subtracted from the three-month test interval results, and (3) the delta risk for the case of challenging event $C$ (Bechtel design) appears to be incorrect (subtracting the one-month case from the three-month case yields $-2.64 \mathrm{E}-8$ rather than the 4.51E-9 that is reported). These comments are minor and will not alter the conclusion that increasing the ESFAS test interval to three months is risk insignificant. 


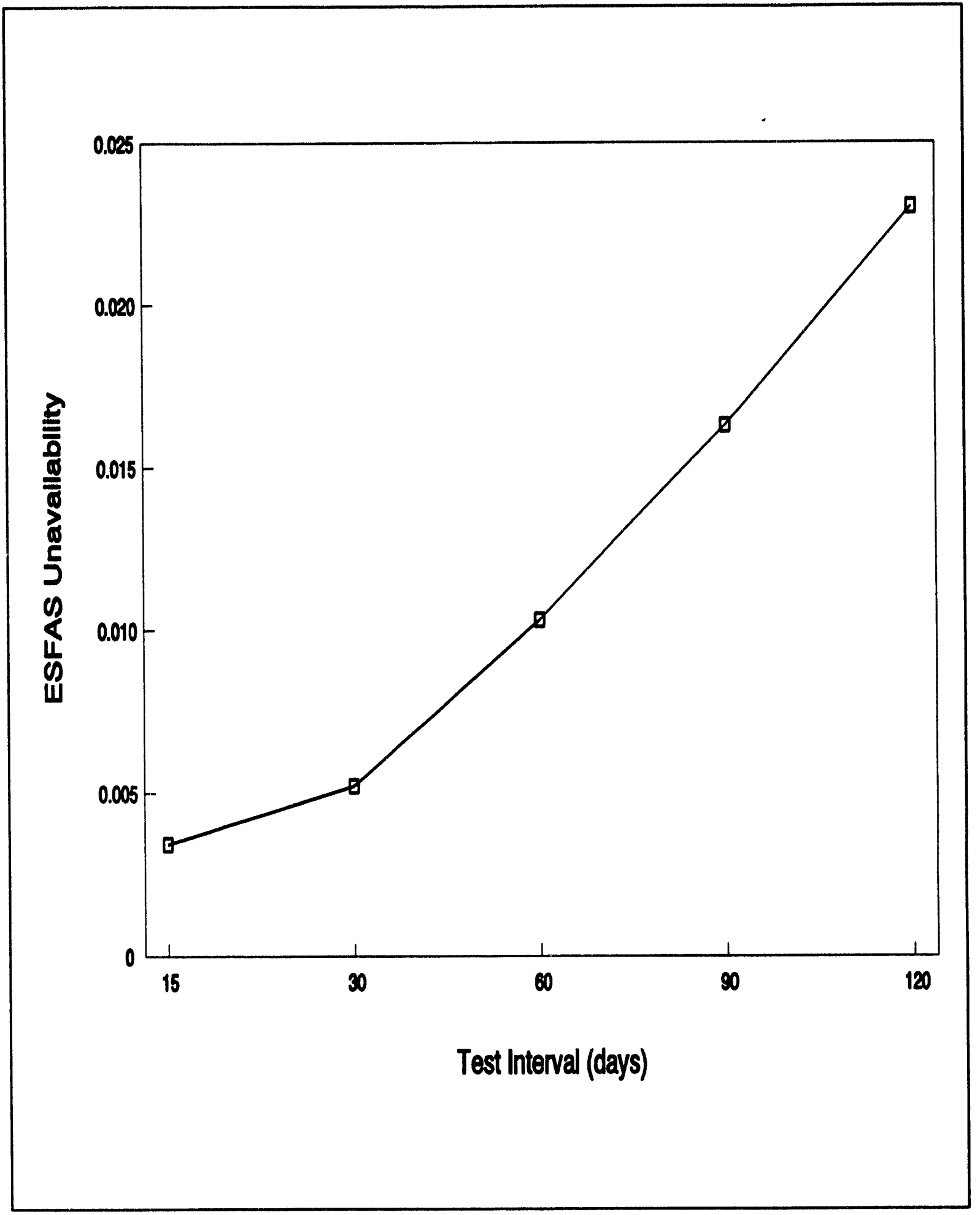

Figure 1. Sensitivity analysis of ESFAS system to test interval changes for FRANTIC calculations. 


\subsection{EVALUATION OF UNCERTAINTY ANALYSIS AND RESULTS}

Chapter 6 of the BAW-10182 report addresses the uncertainty analysis performed on the timeaveraged results. The uncertainty evaluation was performed for each of the three ESFAS designs for (1) each of the six challenging events and (2) an aggregate case including all challenging events. This evaluation was performed for both the current one-month test interval and the proposed threemonth test interval.

One assumption used in the uncertainty analysis was that all of the failure rates had a lognormal distribution and had an error factor of ten. This assumption is valid for the type of analysis that was performed.

Although the failure rates that were used for the analysis did have an appropriate uncertainty associated with them, it appears that neither the challenge event frequency nor the recovery probability parameters were assigned any type of uncertainty. This seems to be an oversight since these two parameters may be much more uncertain and have a greater variability than does the failure rates. While this oversight does not affect the point estimate calculations, it could affect the calculated $95 \%$ upper bound value from the uncertainty analysis. To estimate (approximately) the 95\% upper bound, three NUREG/CR-4550 PRAs ${ }^{11-13}$ were reviewed to see the difference between the point estimate and the upper bound. It was found that the upper bound was typically about a factor of 10 higher than the point estimate. Even though the BAW-10182 analysis did not include the uncertainty on the challenge event frequency and the recovery probabilities, the conclusions of the analysis are still valid since the core melt frequency point estimates are very low (the largest point estimate was $\left.1.45 \times 10^{-7} / \mathrm{yr}\right)$.

While the BAW-10182 report does not go into great detail on how the uncertainty analysis was performed, enough information is given to infer that: (1) the point-estimate ESFAS unavailability equations (from the reliability block diagrams) along with the appropriate initiating events and recovery actions were put into a Monte Carlo code and (2) the Monte Carlo code was used to sample the point-estimate equations 6,000 times.

Performing the uncertainty analysis by using the point-estimate equations will not correctly estimate the uncertainty. Instead, to correctly evaluate the uncertainty for the ESFAS evaluation, the steps that should be taken are: (1) a Monte Carlo code should be used to sample the ESFAS parameters (failure rates, challenge event frequencies, recovery probabilities) for the first iteration, (2) the parameter samples from the Monte Carlo code should be put into the time-dependent analysis code along with the ESFAS equations, (3) the time-dependent code should be run (for both the oneand three-month test intervals) to obtain the time-dependent curve (similar to those in Figure 5-1 through 5-5 in BAW-10182), (4) the time-dependent curve should be integrated to obtain the average risk for the current iteration, (5) the average risk value for the current iteration should be kept in order to perform the statistical analysis, and (6) go back to step (1) and repeat the process a desired number of times (e.g., 6,000). While this process is complicated and calculationally intensive, it will provide a correct uncertainty evaluation for the time-dependent analysis. But, once again the conclusions of the analysis are still valid since the core melt frequency point estimates are very low.

Section 6.2, Uncertainty Analysis Results, of the BAW-10182 report summarizes both the timedependent and uncertainty analysis results. This section contains some minor errors or omissions. These error or omissions are: 
- The second paragraph states that, "The point-estimate value (as calculated by PACRAT)...are given in Table 6-1..." The mean values that are shown in Table 6-1 are actually a pointestimate of the time-averaged results.

- The PDFs shown in Figures 6-4 through 6-6 were obtained by differentiating the cumulative distribution functions (CDFs) shown in Figures 6-1 through 6-3. The reviewer attempted to duplicate the "Total" CDF shown on Figure 6-1 by integrating the PDF shown in Figure 6-4. The CDF that was obtained by integrating the PDF from Figure 6-4 ended up being different than the CDF that was shown in Figure 6-1. The reason for the discrepancy is unknown, but a crude differentiation routine could be to blame. For example, the "dip" at the top of the PDF in Figure 6-4 should show up in the corresponding CDF (i.e., Figure 6-1) as an abrupt change in the slope of the CDF curve. No such change in slope appears on the CDF in Figure 6-1. But, the problems with the PDFs will not change the conclusions of the analysis because the tabulated values and the CDFs appear to be correct. The PDFs are used only for illustrative purposes.

- The first paragraph in Section 6.2 states that, "...it can be asserted with a confidence of at least $95 \%$ that $95 \%$ of a population lies below the $95 \% 195 \%$ value of a random sample from that population." Using the phrase "confidence of at least $95 \%$ " implies that the confidence could actually be higher than $95 \%$ when the confidence is $95 \%$ and cannot be higher without obtaining a new, higher confidence level (and a correspondingly higher value for the incremental core melt frequency).

The cumulative effect of the errors and omissions in the BAW-10182 report will not change the overall conclusions of the report. The point estimate values that were calculated in the BAW-10182 report used appropriate PRA techniques and appear to be correct. Even if it is assumed that a rigorous uncertainty evaluation would provide uncertainty-bounds similar to those found in the NUREG/CR-4550 PRAs, the upper-bound for the risk increase due to increasing the ESFAS test interval would be negligible."

- Assuming the upperbound is a factor of 10 higher than the point estimate gives an upperbound risk-increase due to changing the ESFAS test interval of $1.4 \times 10^{-6} / \mathrm{yr}$, which is 100 times less than the overall mean core damage frequency of $1.1 \times 10^{-4}$ given in the Oconee IPE submittal (Reference 18). 


\subsection{CONCLUSIONS}

In general, this evaluation agrees with the conclusion from the BAW-10182 report that "...the effect on plant risk is insignificant" if the ESFAS test interval is extended to three months from the present one-month interval. The analysis supporting this conclusion incorporated accepted, current practices for risk-based evaluation using PRA methodologies. The analysis evaluated both timedependent and time-independent concerns.

One important area of concern is the issue of instrumentation drift. The issue of drift is not addressed due to the assumption that if a licensee increases the ESFAS test interval they will confirm that instrument drift stays within an acceptable limit. It should be ensured that requirements controlling and measuring instrument drift are in place if the test intervals are extended. Each licensee will need to determine whether the instrument drift over the three-month interval is still bounded by their setpoint calculations. 


\subsection{REFERENCES}

1. R. S. Enzinna, S. H. Levinson, Justification for Increasing the Engineered Safety Features Actuation System (ESFAS) On-Line Test Intervals, BAW-10182, February 1992.

2. J. H. Taylor, B\&W Nuclear Technologies, letter to I. Ahmed, U.S. Nuclear Regulatory Commission, JHT/93-219, September 10, 1993.

3. The Institute of Nuclear Power Operations, NPRDS Reporting Guidance Manual, Revision 04, INPO 83-020B, April 1990.

4. T. Ginzburg and W. E. Vesely, FRANTIC ABC User's Manual, Applied Biomathematics, Inc., November 1990.

5. R. S. Enzinna, S. H. Levinson, and E. W. Swanson, Justification for Increasing the Reactor Trip System On-Line Test Intervals, BAW-10167, May 1986.

6. U.S. Nuclear Regulatory Commission, Safety Evaluation Report, B\&WOG Topical Report BAW10167, Justification for Increasing the RTS On-Line Test Interval, Enclosure in a Letter (NRC Evaluation of BWOG Topical Report BAW-10167 and Supplement 1) from Ashok C. Thadani (NRC) to Courtney W. Smythe (BWOG), December 1988.

7. U.S. Nuclear Regulatory Commission, Reactor Safety Study, WASH-1400 (NUREG-75/014), October 1975

8. A. Mosleh, Procedure for Analysis of Common-Cause Failures in Probabilistic Safety Analysis, NUREG/CR-5801, April 1993.

9. T. R. Meachum and C. L. Atwood, Common Cause Fault Rates for Instrumentation and Control Assemblies, NUREG/CR-3289, May 1983.

10. E. V. Lofgren, M. Thaggard, Analysis of Standby and Demand Stress Failures Modes, NUREG/CR-5823, October 1992.

11. R. C. Bertucio, J. A. Julius, Analysis of Core Damage Frequency: Surry Unit 1 Internal Events, NUREG/CR-4550.

12. M. B. Sattison, K. W. Hall, Analysis of Core Damage Frequency: Zion Internal Events, NUREG/CR-4550.

13. A. M. Kolaczkowski et al., Analysis of Core Damage Frequency: Peach Bottom Unit 2 Internal Events, NUREG/CR-4550. 
14. S. A. Eide, S. V. Chmielewski, and T. D. Swantz, Generic Component Failure Data Base for Light Water and Liquid Sodium Reactor PRAs, EGG-SSRE-8875, February 1990.

15. C. L. Smith, P. G. Ellison, and J. A. Schroeder, An Assessment of the Risk Impact of Integrating Surveillance Testing and Maintenance at Nuclear Power Plants, NUREG/CR-5992.

16. K. D. Russell et al., Integrated Reliability and Risk Analysis System (IRRAS), Version 4.0, Volume 1: Reference Manual, NUREG/CR-5813, EGG-2664, January 1992.

17. P. K. Samanta, S. M. Wong, and J. Carbonaro, Evaluation of Risks Associated with AOT and STI Requirements at the ANO-1 Nuclear Power Plant, NUREG/CR-5200, August 1988.

18. M. S. Tuckman, Duke Power Company, IPE Submittal Report, Oconee Nuclear Station, December 1990. 


